\title{
Change of splittings in Balloon 090100001
} \author{
M. Siwak, ${ }^{1}$ D. Koziet, ${ }^{1}$ S. Zoła ${ }^{1}$

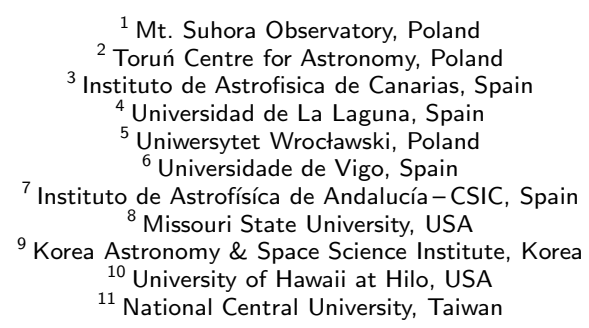

A. Baran, ${ }^{1,2}$ R. Oreiro, ${ }^{3,4}$ A. Pigulski, ${ }^{5}$ F. Pérez, ${ }^{3,4}$ A. Ulla, ${ }^{6}$ R. Garrido, ${ }^{7}$ C. Rodríguez, ${ }^{6,7}$ T. Monserrat, ${ }^{3}$ L. Fox Machado, ${ }^{3}$ J. M. Gonzáles, ${ }^{3}$ M. Reed, ${ }^{8}$ A.-Y. Zhou, ${ }^{8}$ S. Harms, ${ }^{8}$ J. R. Eggen, ${ }^{8}$ S-L. Kim, ${ }^{9}$ R. Crowe, ${ }^{10}$ K-J. Choo, ${ }^{9}$ W-P. Chen, ${ }^{11}$ H-T. Lee, ${ }^{11}$ F-Y. Huan, ${ }^{11}$

\begin{abstract}
We present the first results obtained during the multi-site campaign on the brightest pulsating sdB star Balloon 090100001. Our campaign was carried out in August and September 2005 spanning over 7 weeks. From the frequency analysis we confirm most of frequencies discovered during the 2004 campaign including an equidistant triplet and a quintuplet. The triplet and quintuplet have nearly the same separations, clearly indicating that rotational splitting might be involved. However, the splitting of multiplets increased by about 15\% between 2004 and 2005. As far as we are aware, this is the first example of such a large change of frequency splitting in a pulsating star.
\end{abstract}

\section{The data and results}

The 2005 campaign involved eight optical telescopes. The frequency resolution was around $0.5 \mu \mathrm{Hz}$, similar to that in 2004 data (Baran et al. 2005), but the noise level and aliases were considerably lowered (Baran et al., in preparation).

It appeared from the preliminary analysis of the 2005 data that the amplitudes of some modes change on a time scale of days or weeks. We therefore allowed linear amplitude changes in the analysis. The data were analysed by means of the Fourier transform with consecutive prewhitening of detected frequencies. There were nine modes in the $2.8 \mathrm{mHz}$ region detected: the main mode, the triplet and the quintuplet. Their frequencies derived from the 2004 and 2005 data are schematically shown in Fig. 1. The frequencies of the main mode and the central peak of the triplet did not change between 2004 and 2005. On the other hand, the three frequencies of the quintuplet detected both in 2004 and 2005, presumably with $m=+2,+1$ and -1 , changed their frequencies. However, the multiplets remained almost symmetrical. The average splitting for the triplet increased by about $14 \%$, while for the quintuplet by about $12 \%$ for the $|m|=1$ and by $21 \%$ for the $|m|=2$ components.

\section{What can cause this to happen?}

If we assume that this splitting is caused only by rotation we have to explain how the star increased its rotational frequency by $15 \%$, on average, during a year. Magnetic fields measured 


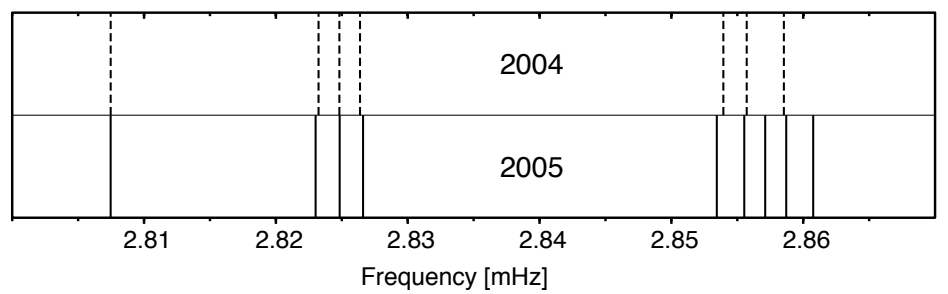

Figure 1: Schematic Fourier spectrum of Balloon 090100001 in the region of the dominant mode.

in a few sdB stars, about $1.5 \mathrm{kG}$ (O'Toole et al. 2005), are too weak to cause this effect. A combination of the rotational splitting modulated by a magnetic field can be a plausible explanation. It seems that this star is really an unusual object among all pulsating sdB stars and monitoring of its pulsational properties is undoubtedly worth doing.

Acknowledgments. This work was supported by the grant 1 P03D 01329.

\section{References}

Baran A., Pigulski A., Kozieł D., et al., 2005, MNRAS, 360, 737

O'Toole S. J., Jordan S., Friedrich S., Heber U., 2005, A\&A, 437, 227

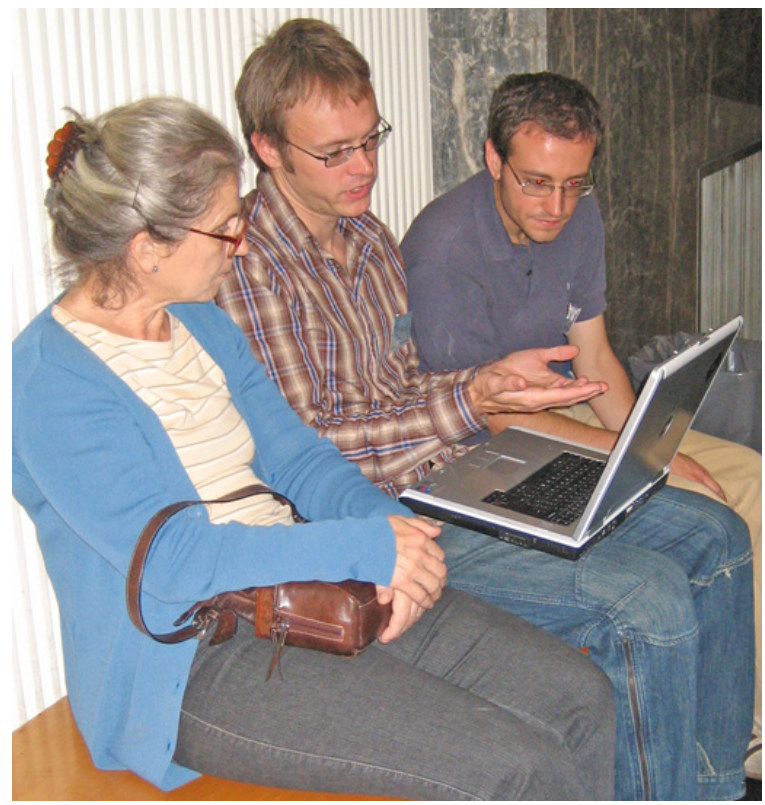

Hans Bruntt has something interesting to show to Tanya Ryabchikova and Luca Fossati. 